\title{
Lohnentwicklung im Lebenszyklus
}

\author{
Eine Analyse von Ausmaß, Begründung und Heterogenität von Lohnsteigerungen
}

\author{
Robert Orlowski · Regina T. Riphahn
}

Angenommen: 22. Februar 2011 / Online publiziert: 22. März 2011

(C) Institut für Arbeitsmarkt- und Berufsforschung 2011

Zusammenfassung Der Beitrag fasst die Ergebnisse einer Reihe von Untersuchungen zusammen, die sich auf Basis der Daten des deutschen Sozio-oekonomischen Panels (SOEP) mit der Lohnstruktur auf deutschen Arbeitsmärkten beschäftigen. Die Analysen nutzen neben Kleinstquadrateschätzern die von Altonji und Shakotko, Rev. Econ. Stud. 54, 437 (1987) und von Topel, J. Polit Econ. 99(1), 145 (1991) verwendeten Schätzverfahren. Diese berücksichtigen die Endogenität der Indikatoren von firmenspezifischer und allgemeiner Arbeitsmarkterfahrung. Der Fokus des Beitrages liegt auf der Analyse möglicher Heterogenitäten in den Lohnstrukturen im privaten und im öffentlichen Sektor, in Ost- und in Westdeutschland, für Männer und Frauen sowie bei Arbeitgebern in großen und kleinen Unternehmen. Die interessantesten Ergebnisse heben deutliche Unterschie-

Diese Arbeit ist im Rahmen des Forschungsprojekts „Lohnentwicklung im Lebenszyklus - Eine Analyse von Ausmaß, Begründung und Heterogenität von Lohnsteigerungen“ im Schwerpunktprogramm der Deutschen Forschungsgemeinschaft (DFG) „Flexibilisierungspotenziale bei heterogenen Arbeitsmärkten“ entstanden. Wir danken dem Herausgeber, einem unbekannten Gutachter und den Teilnehmern der Arbeitstreffen im Schwerpunktprogramm für hilfreiche Kommentare und der Deutschen Forschungsgemeinschaft für die finanzielle Unterstützung. Die in dieser Studie verwendeten Daten des Sozio-oekonomischen Panels (SOEP) wurden vom Deutschen Institut für Wirtschaftsforschung (DIW Berlin), bereitgestellt. Zusätzliche Datengrundlage dieses Beitrags bildet das Querschnittmodell (Version 1) der Linked Employer-Employee Daten des IAB (1998-2007). Der Datenzugang erfolgte über einen Gastaufenthalt am Forschungsdatenzentrum der Bundesagentur für Arbeit im Institut für Arbeitsmarkt- und Berufsforschung (FDZ) und anschließend mittels kontrollierter Datenfernverarbeitung beim FDZ.

R. Orlowski $(\bowtie) \cdot$ R.T. Riphahn

Universität Erlangen-Nürnberg, Lange Gasse 20,

90403 Nürnberg, Deutschland

e-mail: Robert.Orlowski@wiso.uni-erlangen.de de zwischen Ost und West sowie zwischen großen und kleinen Unternehmen hervor.

Schlüsselwörter Lohnstruktur · Humankapital · Firmenzugehörigkeitsdauer · Arbeitsmarkterfahrung

JEL Klassifikationen J31 · J24

Wages over the life cycle-an empirical analysis of wage structures in Germany

Abstract This paper summarizes the findings of studies which investigate the determinants of wages in Germany, using data of the German Socioeconomic Panel (GSOEP). The empirical analyses apply least squares estimates as well as the estimators developed by Altonji and Shakotko, Rev. Econ. Stud. 54, 437 (1987) and Topel, J. Polit Econ. 99(1), 145 (1991). These estimators reduce the endogeneity of indicators of specific and general labor market experience. The studies focus on the heterogeneity in wage determinants in the private and the public sector, in East and West Germany, for men and women, as well as in large versus small firms. Among the most interesting results, we find clear differences in returns to experience between East and West and by employer size.

\section{Einleitung}

Die Entwicklung individueller Kenntnisse und Fertigkeiten ist ein lebensbegleitender Prozess, den Ökonomen als Investitionen in das eigene Humankapital beschreiben. Die erste Investitionsentscheidung wird anhand der Dauer der Schulbildung getroffen (Mincer 1974). Jedes Jahr zusätzlicher Schulbildung führt im späteren Arbeitsleben zu einer durchschnittlichen Lohnsteigerung von rund 6 Prozent 
(Boockmann und Steiner 2006). ${ }^{1}$ Im Arbeitsleben nach der Schulzeit und dem Ende der formalen Ausbildung wird unweigerlich und kontinuierlich zusätzliches Humankapital akkumuliert. Zu unterscheiden sind hier zwei Arten von $\mathrm{Hu}-$ mankapital (Becker 1975): allgemeines Humankapital, welches durch aktive Partizipation am Arbeitsmarkt angesammelt wird und firmenspezifisches Humankapital, welches etwa anhand firmenspezifischer Kenntnisse (zum Beispiel firmeninterne Software, Firmenstrukturen und -netzwerke) erworben wird. Firmenspezifisches Humankapitals ist immobil und geht, im Gegensatz zum allgemeinen Humankapital, verloren, wenn Arbeitnehmer ihren Arbeitgeber wechseln. Höheres Humankapital führt im Normalfall zu höherer Produktivität am Arbeitsmarkt und somit zu höheren Löhnen.

Individuelles wie gesamtgesellschaftliches Lohnwachstum ist wirtschaftspolitisch bedeutsam und eine Kenntnis der Lohnstrukturen ist in vielen Bereichen relevant. So lässt sich zum Beispiel anhand der Humankapitalrenditen evaluieren, ob berufliche Fort- und Weiterbildung zu produktivitätsbedingtem Lohnwachstum führt. Gleichzeitig lassen sich die Kosten von Erwerbsunterbrechungen (entgangenes Lohnwachstum) und von Arbeitsplatzmobilität (Verlust an firmenspezifischem Humankapital) analysieren.

Grundsätzlich kann ein geringes gesamtgesellschaftliches Lohnwachstum ein Hinweis auf die sinkende relative Bedeutung des Produktionsfaktors Arbeit in der Volkswirtschaft sein. Sinkende Arbeitseinkommensquoten (AEQ) ${ }^{2}$ können, abgesehen von kurzfristig verzögerter Beschäftigungsanpassung, ein Hinweis darauf sein, dass die Erträge der Faktoren Arbeit und Kapital immer mehr zu Ungunsten der Arbeitnehmer verteilt werden. Dieses mögliche Ergebnis von Lohnzurückhaltung wird insbesondere von Gewerkschaften stark kritisiert.

Deutschland genießt als größte Volkswirtschaft Europas besonderes wissenschaftliches Interesse. Der deutsche Arbeitsmarkt der letzten Jahre zeichnet sich im europaweiten Vergleich durch sein geringes Lohnwachstum aus (Statistisches Bundesamt 2010a). So sank im letzten Jahrzehnt die AEQ stetig (BMF 2010) ${ }^{3}$. Durch das geringe Lohnwachstum erwirtschaftete sich Deutschland einerseits einen Kostenvorteil gegenüber anderen, durch hohes Lohnwachstum

\footnotetext{
${ }^{1}$ Die Höhe der Bildungsrendite fällt je nach betrachteter Geburtskohorte unterschiedlich aus. Der hier genannte Wert bezieht sich auf Männer ab Geburtskohorte 1960 und Frauen ab Geburtskohorte 1965 auf Basis der Daten des sozio-oekonomischen Panels.

${ }^{2}$ Die AEQ spiegelt die relative Bedeutung des Faktors Arbeit in der funktionalen Einkommensverteilung auf Arbeit und Kapital wider.

${ }^{3}$ Die Analysedaten beziehen sich auf die Jahre bis 2008. Erste Prognosen für 2009 deuten aufgrund starker Einbrüche der Unternehmensund Vermögenseinkommen auf eine gestiegene AEQ hin. Änderungen in der AEQ werden vor allem auf Änderungen in der Sektorstruktur zurückgeführt.
}

gekennzeichneten Ländern Europas. Andererseits führt ein geringes Lohnwachstum möglicherweise zu Einbußen im privaten Konsum und zu niedrigeren Einnahmen aus Steuern und Sozialversicherungsbeiträgen.

Deutschland bietet für die Analyse von Lohnstrukturen einen weiteren interessanten Aspekt: die Möglichkeit, die Entwicklung in den beiden historisch getrennten Arbeitsmärkte in Ost- und Westdeutschland zu verfolgen, die nun seit 20 Jahren wiedervereinigt sind. Der Osten - das ,Süditalien“ Deutschlands - stellt auch heute trotz massiver öffentlicher Transferzahlungen noch keine einheitlich „blühende Landschaft" dar (Berlemann und Thum 2005; Heilemann 2005). Verglichen mit Westdeutschland, ist der Osten Deutschlands gekennzeichnet durch niedrigere Durchschnittslöhne, höherer Arbeitslosigkeit und einem schleppenden wirtschaftlichen Aufholprozess. Die Firmenlandschaft ist überwiegend geprägt von kleinen und mittelgroßen Firmen, geringer Tarifbindung, niedrigem Innovationsgrad und mangelnder Vernetzung (Uhlig 2008; Eickelpasch 2009; Kuehn 2010; Fitzenberger et al. 2011). Gleichzeitig liegt die Arbeitsmarktpartizipation von Frauen in Ostdeutschland deutlich über westdeutschem Niveau, wodurch das Arbeitskräftepotential besser ausgenutzt wird.

In dieser Zusammenfassung unserer Studien (Orlowski und Riphahn 2008, 2009; Orlowski 2011a, 2011b) ${ }^{4}$ beschreiben wir die Determinanten der Löhne von Arbeitnehmern in Deutschland. Im Fokus stehen dabei die Lohnentwicklung im Lebenszyklus sowie die relative Bedeutung der beiden Humankapitalarten. Die große Herausforderung bei der Bewertung der Bedeutung des Humankapitals für die Löhne liegt darin, die Höhe der Rendite der Firmenzugehörigkeitsdauer (firmenspezifisches Humankapital) und der Rendite der allgemeinen Arbeitsmarkterfahrung (allgemeines Humankapital) verlässlich zu bestimmen und die kausalen Effekte auf den Lohn zu identifizieren.

Unsere Analyse der Lohnstrukturen umfasst vier Dimensionen: Wir untersuchen die Unterschiede in den Humankapitalrenditen (a) im öffentlichen und privaten Sektor, (b) in Ost- und Westdeutschland, (c) zwischen den Geschlechtern und (d) in Bezug auf unterschiedliche Firmengrößen. Anhand dieser vier Dimensionen diskutieren wir im Folgenden die Literatur, unsere Ergebnisse und die wirtschaftspolitischen Implikationen der Befunde.

\section{Literatur, Relevanz und Hypothesen}

Die Höhe der Humankapitalrenditen beschäftigt Ökonomen seit langem. Von Interesse ist, welcher Anteil am Lohn-

\footnotetext{
${ }^{4}$ In diesem Beitrag wird bewusst auf technische Details verzichtet. Es sei an dieser Stelle auf die ausführliche Beschreibung in den einzelnen Studien verwiesen.
} 
wachstum der Akkumulation von firmenspezifischem $\mathrm{Hu}$ mankapital zugeschrieben werden kann und welcher Anteil sich auf die Akkumulation von allgemeinem Humankapital zurückführen lässt. Bei der Berechnung der Humankapitalrenditen führt die klassische KleinstequadrateMethode (KQ) aufgrund der Endogenität der allgemeinen Arbeitsmarkterfahrung und der Firmenzugehörigkeitsdauer zu verzerrten Schätzergebnissen. Die Arbeitsmarkterfahrung ist endogen, wenn beispielsweise hochmotivierte Personen mehr Arbeitsmarkterfahrung aufweisen und gleichzeitig diese Motivation auch zu höheren Löhnen führt. Die Firmenzugehörigkeit ist endogen, wenn die ArbeitgeberArbeitnehmer-Passung (job match) besonders hoch ist und dies gleichzeitig zu einem längeren Arbeitsverhältnis und zu höheren Löhnen führt.

Die Diskussion über die relative Bedeutung der beiden Humankapitalarten wurde Ende der 1980er und zu Beginn der 1990er Jahre durch die richtungsweisenden Studien von Altonji und Shakotko (fortan AS 1987) und Topel (1991) intensiviert. Beide Studien stellen sich der Herausforderung, die kausalen Renditen des Humankapitals zu identifizieren, wobei sie methodisch verschiedene Ansätze verwenden: AS verwenden Instrumentalvariablen (IV) und Topel ein zweistufiges Verfahren (2SFD). Die Autoren kommen zu unterschiedlichen Ergebnissen. So finden AS nahezu keine Rendite des firmenspezifischen Humankapitals und schreiben somit der Akkumulation von allgemeinem Humankapital den Großteil des Lohnwachstums zu. Topel hingegen findet sehr hohe Renditen der Firmenzugehörigkeit und schreibt rund 40 Prozent des Lohnwachstums im jeweiligen Beschäftigungsverhältnis der Akkumulation von firmenspezifischem Humankapital zu.

Beide Schätzverfahren sind nur unter starken Annahmen konsistent. Dennoch kann man aus dem Vergleich der Schätzergebnisse neue Erkenntnisse gewinnen, da diese als obere und untere Grenzen der wahren Renditen der Firmenzugehörigkeit interpretiert werden können. ${ }^{5}$ Sie gehören auch heute noch zum internationalen Standardinstrumentarium in der Analyse von Lohnstrukturen (siehe zum Beispiel Dustmann und Pereira 2008; Williams 2009; Buchinsky et al. 2010).

\footnotetext{
${ }^{5}$ Die Schätzverfahren erzeugen nur unter der Annahme, dass ein Jobwechsel zufällig ist, unverzerrte Schätzergebnisse. Diese Annahme ist vor dem Hintergrund der Such- und Matchingtheorien (Burdett 1978; Jovanovic 1979) kaum haltbar. Die berechneten Senioritätsrenditen sind bei beiden Schätzverfahren aufgrund unbeobachtbarer Job-MatchHeterogenität nach unten verzerrt. Die Senioritätsrendite im 2SFDVerfahren ist zusätzlich durch individuelle Heterogenität nach oben verzerrt (Altonji und Williams 2005). Wenn die positive Verzerrung die negative überkompensiert, ergibt sich eine obere Grenze der Senioritätsrendite. Topel (1991) selbst sieht die nach seiner Methode ermittelten Senioritätsrendite als untere Grenze an. Für eine weiterführende Diskussion der identifizierenden Annahmen und Ausmaß und Richtung der Verzerrungen siehe zum Beispiel Altonji und Williams (2005).
}

Unsere Analysen der Heterogenität von Humankapitalrenditen unterscheiden vier Dimensionen. Im Folgenden motivieren wir jeweils die wirtschaftspolitische Relevanz der Fragestellung, fassen den zugrundliegenden Stand der Literatur zusammen und formulieren zentrale, empirisch zu überprüfende Hypothesen.

Die erste Dimension unserer Analyse betrachtet die Lohnunterschiede im öffentlichen und privaten Sektor. Der öffentliche Sektor ist wirtschaftspolitisch von großem Interesse. Zum einen handelt es sich um den größten Arbeitgeber in Deutschland. ${ }^{6}$ Zum anderen hat die Lohnsetzung im öffentlichen Sektor Einfluss auf die Löhne in der Privatwirtschaft. Ein großer Lohnunterschied zwischen Privatwirtschaft und öffentlichem Sektor (public sector wage gap) kann den Druck auf die Löhne der Privatwirtschaft erhöhen (spillover effect), die Wettbewerbsfähigkeit in der Privatwirtschaft gefährden und zusätzlich inflationssteigernde Wirkung haben (Melly 2005). Gleichzeitig sind die Löhne im öffentlichen Sektor weniger flexibel, was insbesondere in wirtschaftlichen Krisen zu immensen Kosten führen kann und somit fiskalpolitisch bedeutend ist.

Unser Beitrag über die Lohnstrukturen im öffentlichen und privaten Sektor (Orlowski und Riphahn 2008) knüpft an die Erkenntnisse dreier empirischer Studien an: (i) Dustmann und Meghir (2005) untersuchen die Humankapitalrenditen für eine Stichprobe junger Männer. Die Autoren finden sehr hohe Renditen der Firmenzugehörigkeit von 2,5 bis 4 Prozent pro Jahr. (ii) Dustmann und Pereira (2008) betrachten in ihrer Analyse Männer bis Alter 60 und finden deutlich geringere Renditen der Firmenzugehörigkeitsdauer (2 Prozent bei 10 Jahren Firmenzugehörigkeit). (iii) Die Studie von Amann und Klein (2007) hingegen ergibt rund 1 Prozent Lohnwachstum mit jedem zusätzlichen Jahr Firmenzugehörigkeit. Diese Autoren stellen fest, dass individuelle Heterogenität eine treibende Kraft für das Lohnwachstum ist. Alle drei Studien betrachten den Arbeitsmarkt im privaten Sektor Westdeutschlands. In unserer Studie untersuchen wir, erstmals für Deutschland, zusätzlich zum privaten auch den öffentlichen Sektor und testen die Hypothese gleicher Lohnstrukturen im öffentlichen und privaten Bereich. Die bisher vorliegenden Studien zum öffentlichen Sektor in Deutschland (Dustmann und van Soest 1997; Melly 2005; Heitmueller und Mavromaras 2007) betrachteten nicht die Lohnstrukturen, sondern interessieren sich für die Selektion der Arbeitnehmer in den jeweiligen Sektor. Ebenso wenig wurde bisher Ostdeutschland bei der Analyse der Lohnstrukturen berücksichtigt.

Die zweite Dimension unserer Analyse der Lohnstrukturen betrachtet die Unterschiede auf den zwei regionalen Arbeitsmärkten in Ost- und Westdeutschland. Vor dem Hin-

${ }^{6} 13,3 \%$ der Arbeitnehmer bzw. 4,6 Mio. Personen waren im Jahr 2005 im öffentlichen Sektor beschäftigt (Statistisches Bundesamt 2010b). 
tergrund der immer noch dominierenden strukturellen Unterschiede zwischen den Arbeitsmärkten in Ost- und Westdeutschland ist es aus wirtschaftspolitischer Perspektive bedeutsam, mehr über die den Lohnstrukturen unterliegenden Mechanismen zu erfahren, um gegebenenfalls steuernd eingreifen zu können. Hierzu bieten sich viele verschiedene Ansatzpunkte an; unser Beitrag konzentriert sich auf einen Vergleich der Humankapitalrenditen in den beiden regionalen Arbeitsmärkten.

Die frühe und noch optimistische nationale Literatur aus den Jahren kurz nach der Wiedervereinigung prognostizierte eine schnelle Anpassung des ostdeutschen Arbeitsmarktes an westdeutsche Strukturen. Im Einklang mit dem in der ehemaligen DDR dominanten Gedanken der Lohngleichheit, zeigen Bird et al. (1994), dass die Heterogenität der Löhne in der DDR deutlich geringer war als im westdeutschen Arbeitsmarkt, und dass die Renditen des Humankapitals vor der Wiedervereinigung in Ostdeutschland etwa halb so hoch waren wie die in Westdeutschland. Erste ernüchternde Ergebnisse zur tatsächlichen Anpassung der Lohnstrukturen lieferte die Studie von Jurajda und Harmgart (2007). Die Autoren benutzen Daten bis 1995 und finden noch 5 Jahre nach der Wiedervereinigung ein flaches Lohnprofil im Lebenszyklus für Ostdeutschland. Das flache ostdeutsche Lohnprofil wurde auch noch 10 Jahre nach der Wiedervereinigung von Franz und Steiner (2000) und Gathmann (2005) bestätigt.

Die internationale Literatur für Transformationsländer findet bei der Analyse von Lohnstrukturen in ehemalig sozialistischen Ländern nur ein schwaches Lohnwachstum bei zunehmender Firmenzugehörigkeit und steigender allgemeiner Arbeitsmarkterfahrung (zum Beispiel Jones und Simon 2005 für Bulgarien, Münich et al. 2005 für Tschechien, Noorkoiv et al. 1997 für Estland). Die Analyse Deutschlands bietet gegenüber anderen Transformationsländern einen Vorteil: Die Entwicklung Ostdeutschlands kann vergleichend mit der Entwicklung des marktwirtschaftlichen System Westdeutschlands analysiert werden, da sich beide Teile Deutschlands Sprache, Geschichte und seit 1990 auch die Arbeitsmarktinstitutionen teilen. Unsere Studie (Orlowski und Riphahn 2009) schließt eine Lücke in der Literatur und untersucht das Lohnwachstum in Ost- und Westdeutschland unter Berücksichtigung der potentiellen Endogenität der Humankapitalrenditen. Wir testen die Hypothese, dass die Lohnstrukturen in Ostdeutschland knapp zwei Jahrzehnte nach der Wiedervereinigung mit denen in Westdeutschland vergleichbar sind.

Die dritte Dimension unserer Analyse untersucht die Lohnunterschiede zwischen den Geschlechtern (gender wage gap). Das Thema genießt hohe politische und ökonomische Aufmerksamkeit, da sich die Bedeutung weiblicher Erwerbstätiger am Arbeitsmarkt in den letzten Jahrzehnten deutlich verändert hat. Neben dem steigenden all- gemeinen Bildungsniveau von Frauen beobachtet man eine höhere Arbeitsmarktpartizipation und eine zunehmende Konvergenz der Lohn- und Beschäftigungsstrukturen zwischen Männern und Frauen (O'Neill und Solomon 1993; Blau und Kahn 1994). Weltweit fielen die Lohnunterschiede zwischen den Geschlechtern zwischen 1960 und 1990 von 65 auf 30 Prozent. Gleichzeitig blieb der unerklärte Teil des gender wage gap im Beobachtungszeitraum stabil bei rund 20 Prozent (Weichselbaumer und Winter-Ebmer 2005). Weichselbaumer und Winter-Ebmer (2005) finden in ihrer umfangreichen Meta-Analyse, dass die Humankapitalausstattung einen wichtigen Beitrag zur Erklärung der Lohnunterschiede zwischen den Geschlechtern liefert.

Die Literatur bietet vielfältige Ansätze zur Analyse der Lohnunterschiede zwischen Männern und Frauen (Altonji und Blank 1999). Häufig werden diese anhand von Unterschieden in den Partizipationsraten, dem Zeitpunkt von Erwerbsunterbrechung und unterschiedlicher Arbeitsplatzmobilität erklärt (Light und Ureta 1990; Filer 1993; Wellington 1993; Kim und Polachek 1994; Munasinghe et al. 2008). ${ }^{7}$

In der ökonomischen Theorie gibt es konkurrierende Modelle, die entweder niedrigere oder höhere Renditen der Firmenzugehörigkeit für Frauen vorhersagen. Die klassische Humankapitaltheorie (Becker 1962) ist „geschlechtsblind“. Unter Annahme von Abschreibungen des Humankapitals während der Erwerbsunterbrechungen (Mincer und Polachek 1974), würde man im Mittel niedrigere Renditen des Humankapitals für Frauen erwarten. ${ }^{8}$

Hashimoto's (1981) efficiency cost sharing Theorie sagt höhere Renditen für Frauen als für Männer voraus: ${ }^{9}$ Je höher der Eigenanteil an den Kosten firmenspezifischer Investitionen, desto steiler ist das erwartete Lohnprofil, da diese Investitionen nur in der jeweiligen Firma von Nutzen sind und diese sich möglichst schnell amortisieren sollten. Da Frauen aufgrund von Familienarbeit als zusätzlicher Option außerhalb des Arbeitsmarktes eine kürzere erwartete Verweildauer im Unternehmen haben, ist der Eigenanteil der Kosten firmenspezifischer Investitionen höher als bei Männern. Somit sollten bei Frauen höhere Renditen des firmenspezifischen Humankapitals zu beobachten sein.

\footnotetext{
${ }^{7}$ Die Literatur über (statistische) Diskriminierung wird an dieser Stelle nicht ausführlich diskutiert. Ein Überblicksartikel ist zum Beispiel Altonji und Blank (1999).

${ }^{8}$ Die Abschreibungen kommen zum einen durch einen Verfall der bisherigen Fähigkeiten (Humankapitalstock) und zum anderen durch mangelnde Neuinvestitionen (zum Beispiel in Reaktion auf technologischen Fortschritt) zustande. Wenn der Lohn ein Maß der Produktivität ist, führen Humankapitalabschreibungen während der Erwerbsunterbrechung zu niedrigeren Löhnen bei Wiedereintritt in den Arbeitsmarkt. Bei gegebener Firmenzugehörigkeitsdauer (aber unterschiedlichem Alter) führt dies zu einem geringeren durchschnittlichen Lohnwachstum, welches sich in den Humankapitalrenditen widerspiegelt.

${ }^{9}$ Hashimoto's (1981) Theorie wurde erstmals von Becker und Lindsay (1994) getestet und nicht verworfen.
} 
Ebenso wie die ökonomische Theorie, präsentiert sich auch die internationale empirische Literatur uneinig. Unklar ist, ob die Renditen des Humankapitals für weibliche Beschäftigte höher (zum Beispiel Banzhaf 2005), niedriger (zum Beispiel Kim und Polachek 1994) oder identisch mit denen für Männer (zum Beispiel Erosa et al. 2002) sind. Die bisherige deutsche Literatur betrachtet zumeist in Anlehnung an die Diskussion bei Goldin (1986) die Art der Entlohnung als Ursache für unterschiedliche Renditen des Humankapitals (Heywood und Jirjahn 2002; Jirjahn und Stephan 2004) oder verwendet nicht repräsentative Daten einzelner großer Unternehmen (Pfeifer und Sohr 2008).

Ein Problem bei der Analyse der Lohnstrukturen weiblicher Beschäftigter ist ihr Partizipationsverhalten, da die Erwerbstätigkeit aufgrund von Geburten und Pflegetätigkeiten häufiger von Unterbrechungen betroffen ist. Um die Wirkung der potentiell verzerrenden Selbstselektion in die Erwerbstätigkeit und einer haushaltsspezifisch-gemeinsamen Arbeitsangebotsfunktion von Ehepartnern abzuschätzen, dehnt die internationale Literatur die Analyse auf Familien mit und ohne Kinder aus (family gap nach Waldfogel 1998). Der von Waldfogel (1998) gefundene familienbedingte Unterschied sollte zwischen kinderlosen Frauen und (kinderlosen) Männern nicht zu finden sein. Für den deutschen Raum gibt es bisher keine Studie, die die relative Bedeutung von allgemeiner und firmenspezifischer Arbeitsmarkterfahrung für beide Geschlechter unter Berücksichtigung der potentiellen Endogenität dieser Lohnstrukturdeterminanten analysiert. Unsere Studie (Orlowski 2011a) überprüft die Hypothese, ob bei Frauen höhere Renditen der Firmenzugehörigkeit beobachtbar sind und somit Hashimotos efficiency cost sharing Theorie Bestätigung findet. ${ }^{10}$

Die vierte Dimension unserer Analyse untersucht die Lohnunterschiede in großen und kleinen Unternehmen. Die bisherige Literatur widmet der Tatsache große Aufmerksamkeit, dass größere Firmen einen höheren Lohn zahlen. Dies wird u.a. mit den angebotenen Arbeitskonditionen, höherer Qualität der nachgefragten Arbeit und unterschiedlichen Kosten der Überwachung begründet (Mellow 1982; Garen 1985; Brown und Medoff 1989). Wir verbreitern den Ansatz, der bislang nach firmengrößenspezifischen Unterschieden im Lohnniveau fragt und prüfen, ob es darüber hinaus Unterschiede in den Lohnstrukturen gibt und sich etwa die Humankapitalrenditen zwischen Arbeitgebern verschiedener Größe unterscheiden. Auf diesem Wege stellen

\footnotetext{
${ }^{10}$ Die Selbstselektion der Frauen in Berufe mit flachen Lohnprofilen, aufgrund des kürzer zu erwartenden Erwerbslebens (Polachek 1981) oder höheren Jobfluktuationen (Salop und Salop 1976), bleibt in unserer Analyse weitestgehend unberücksichtigt. Die geschlechterspezifische berufliche Mobilität kommt als weitere Quelle für Lohnunterschiede in Betracht (vgl. zum Beispiel Fitzenberger und Kunze (2005) für Deutschland).
}

wir neue Evidenz zur Verfügung, die auch wirtschaftspolitischen Entscheidungsträger hilfreich sein kann, um diese erheblichen Lohndifferenzen am Arbeitsmarkt zu verstehen. Immerhin ist der Lohnunterschied zwischen großen und kleinen Firmen substantiell; er ist vergleichbar mit dem gender wage gap und sogar größer als Lohnunterschiede zwischen schwarzen und weißen Amerikanern (Idson und Oi 1999).

In der Modellwelt der deferred compensation nach Lazear (1979) sind Senioritätslöhne als langfristiger Motivationsanreiz besonders in großen Firmen plausibel, da hier individuelle Überwachungsmechanismen nicht so gut greifen wie in kleinen, überschaubaren Unternehmen. Bisher konnten durch individuelle Charakteristika der Arbeitnehmer nur ein kleiner Teil ihrer Lohnunterschiede erklärt werden. Die Literatur verweist darauf, dass für die Messung zentraler Firmenmerkmale keine Daten bereit stehen. Troske (1999) verwendete erstmals individuelle und zusätzlich firmenspezifische Merkmale in seiner Analyse und stellte fest, dass auch auf diese Weise noch 55 Prozent des Lohnunterschieds unerklärt blieben. Neuere Literatur untersucht das Mobilitätsverhalten der Arbeitskräfte in kleinen und großen Firmen als möglichen Erklärungsansatz für Lohnunterschiede (Farber 1999; Buchinsky et al. 2010). Bagger et al. (2006) und Yamaguchi (2010) zeigen jedoch, dass die Humankapitalausstattung der Arbeitskräfte eine größere Rolle spielt als deren Suchverhalten. In unserer Studie überprüfen wir die Hypothese, dass steilere Lohnprofile in großen Firmen beispielsweise aufgrund höherer Kosten der Überwachung oder als motivationssteigernde Anreizmechanismen im Sinne von Lazear (1979) eingesetzt werden.

\section{Ergebnisse}

\subsection{Lohnstrukturen im öffentlichen und privaten Sektor}

Auf Basis von Daten des deutschen Sozio-oekonomischen Panels (SOEP) zeigen die Ergebnisse unserer Studie (Orlowski und Riphahn 2008), dass es Unterschiede in den Humankapitalrenditen zwischen dem privaten und dem öffentlichen Sektor gibt. ${ }^{11}$ Die Renditen der allgemeinen Arbeitsmarkterfahrung variieren. So gehen 10 Jahre allgemeine Arbeitsmarkterfahrung im Privatsektor mit 35 Prozent Lohnwachstum einher und im öffentlichen Sektor mit 55 Prozent. Die Renditen der Firmenzugehörigkeit sind jedoch

\footnotetext{
${ }^{11}$ Die Firmenzugehörigkeitsdauer wird im Fragebogen unabhängig vom Arbeitgeber über zwei Fragen bestimmt: „Haben Sie nach dem 31. Dezember 20xx die Stelle gewechselt oder eine Arbeit neu begonnen?“ Eine mögliche Antwort lautet: „Habe innerhalb des Unternehmens die Stelle gewechselt“. Falls letzteres angegeben wurde, wurde kein Jobwechsel ausgewiesen und die Firmenzugehörigkeitsdauer setzt sich fort.
} 


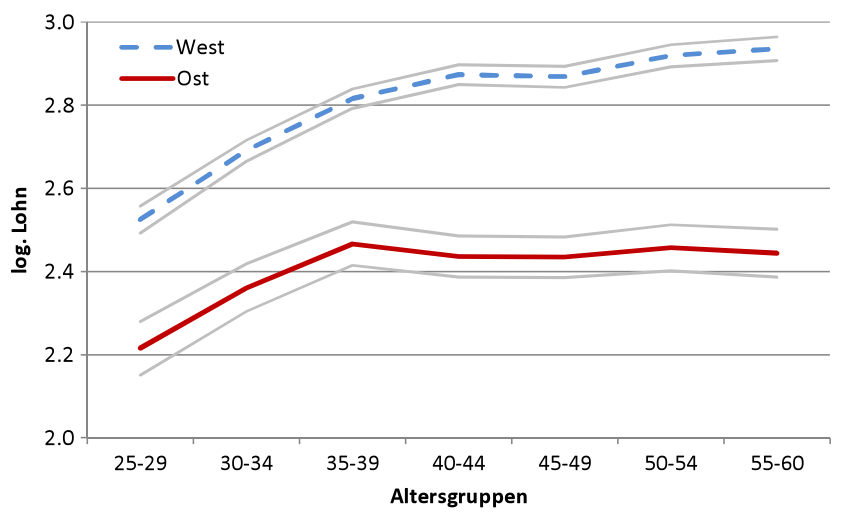

Abb. 1 Lohnstrukturen im Lebenszyklus in Ost- und Westdeutschland. Quelle: SOEP, 2002-2006, eigene Berechnungen. Die Ergebnisse basieren auf einer Regression mit Altersgruppen und Jahresindikatoren. Die 95 \% Konfidenzbänder sind durch graue Linien dargestellt

nach Berücksichtigung der potentiellen Endogenität der Firmenzugehörigkeitsdauer nach der Methode von AS (1987) in beiden Sektoren sehr niedrig. Es zeigt sich, dass das Lohnwachstum durch längere Verweildauer beim Arbeitgeber ökonomisch kaum relevant ist und sich nicht systematisch zwischen den beiden Sektoren unterscheidet. ${ }^{12}$ Die Ergebnisse sind robust gegenüber alternativen Spezifikationen der funktionalen Form. Insofern gibt es keine Anhaltspunkte für wirtschaftspolitische Maßnahmen zum Abbau von sektorenspezifischen Lohnrigiditäten, für segmentierte Arbeitsmärkte oder für ein ungenutztes Flexibilisierungspotenzial aufgrund einer unterschiedlichen, senioritätsbedingten Entlohnungsstruktur im öffentlichen oder privaten Sektor.

\subsection{Ergebnisse für Ost- und Westdeutschland}

Bei der Analyse der Arbeitsmärkte in Ost- und Westdeutschland (Orlowski und Riphahn 2009) offenbarten sich überraschend deutliche Unterschiede in den Lohnstrukturen auch knapp zwei Jahrzehnte nach der Wiedervereinigung. Das Lohnwachstum aus allgemeiner Arbeitsmarkterfahrung ist in Ostdeutschland sehr gering. Abbildung 1 beschreibt den mittleren logarithmierten Lohnsatz für sieben Altersgruppen in Ost- und Westdeutschland. Die regionalen Unterschiede sowohl in der absoluten Lohnhöhe als auch im Lohnwachstum werden unmittelbar deutlich. Die Resultate unserer Schätzungen bestätigen die unterschiedlichen Renditen der Arbeitsmarkterfahrung.

Die Regressionsergebnisse zeigen, dass die Renditen der Firmenzugehörigkeit in Ost- und Westdeutschland gering sind (rund 1 Prozent Lohnwachstum pro Jahr in den ersten

\footnotetext{
${ }^{12}$ Unsere Spezifikation der Lohngleichung berücksichtigt neben der Firmenzugehörigkeitsdauer und der tatsächlichen Arbeitsmarkterfahrung, das Bundesland, Industriesektoren, Familienstand, Firmengröße, Jahr und Bildung (Anzahl der Schuljahre).
}

fünf Jahren) und sich nicht systematisch unterscheiden. ${ }^{13}$ Die Renditen der allgemeinen Arbeitsmarkterfahrung sind in Ostdeutschland, verglichen mit denen in Westdeutschland, sehr niedrig: 10 Jahre Arbeitsmarkterfahrung führen in Westdeutschland zu einem Lohnwachstum zwischen 34 und 43 Prozent. In Ostdeutschland hingegen ist nur ein Lohnwachstum von 22 bis 27 Prozent zu beobachten, welches nicht statistisch signifikant von Null verschieden ist. ${ }^{14}$ Eine Analyse der Unterschiede in den Renditen zwischen Bevölkerungsgruppen mit unterschiedlichen formalen Bildungsabschlüssen zeigt, dass die Unterschiede zwischen Ost- und Westdeutschland mit zunehmendem Bildungsgrad zunehmen. Nachdem in der Literatur häufig das Erodieren des im sozialistischen Regime erworbenen Humankapitals als Hauptursache für den flachen Lohnverlauf in Ostdeutschland herangezogen wird, betrachten wir junge Personen, die erst ab 1990 in den Arbeitsmarkt eingetreten sind und somit ihr gesamtes arbeitsmarktbezogenes Humankapital im marktwirtschaftlichen System akkumuliert haben. Auch in diesem Fall bestätigt sich der flache Lohnverlauf in Ostdeutschland. Diese Ergebnisse schließen ein nach der Wiedervereinigung veraltetes Humankapital als Erklärungsansatz aus, da ja nur solche Personen betrachtet wurden, die vor 1990 kein Humankapital am Arbeitsmarkt erworben hatten.

Zwei mögliche Erklärungsansätze kommen für die im Vergleich zu Westdeutschland geringeren Renditen des allgemeinen Humankapitals in Ostdeutschland in Frage: Entweder es handelt sich um einen Preiseffekt, durch den das homogene Gut „Arbeitsmarkterfahrung“ in Ost und West regional unterschiedlich bewertet wird. Alternativ ist es möglich, dass die in Ost- und Westdeutschland erworbene Arbeitsmarkterfahrung von unterschiedlicher Qualität ist, so dass unterschiedliche Bewertungen am Arbeitsmarkt zu erwarten sind. Um dies zu überprüfen, untersuchten wir die Renditen der Arbeitsmarkterfahrung von Personen, die vor der Wiedervereinigung in Ostdeutschland wohnhaft waren und heute auf dem westdeutschen Arbeitsmarkt tätig sind. Es zeigte sich, dass diese Personen geringere Renditen der Arbeitsmarkterfahrung erzielen als vergleichbare westdeutsche Arbeitskräfte. Umgekehrt erhalten Personen, die vor der Wiedervereinigung in Westdeutschland und heute auf

\footnotetext{
${ }^{13}$ Die niedrigen Renditen der Firmenzugehörigkeit in Deutschland sind im Einklang mit der Literatur, siehe z. B. Schönberg (2007) für eine Analyse westdeutscher Männer mit Daten der IABBeschäftigtenstichprobe bis 1994.

${ }^{14}$ Zusätzliche Kontrollvariablen in unserer Spezifikation der Lohngleichung sind: höchster Bildungsabschluss, Industriesektoren, Immigrationsstatus, Bundesland und Jahr. Vielfältige Tests bestätigen die Robustheit dieser Ergebnisse. So wurden zum einen verschiedene Firmengrößenindikatoren genutzt und zum anderen verschiedene Spezifikationen der funktionalen Form des Schätzmodells verwendet.
} 
Tab. 1 Vorhergesagte Renditen des Humankapitals für Männer und Frauen in Deutschland

\begin{tabular}{|c|c|c|c|c|c|c|c|}
\hline \multirow[t]{3}{*}{ Schätzverfahren } & & \multicolumn{3}{|l|}{ Frauen } & \multicolumn{3}{|l|}{ Männer } \\
\hline & & (1) & (2) & (3) & (4) & (5) & (6) \\
\hline & & OLS & AS & 2SFD & OLS & AS & 2SFD \\
\hline \multirow{6}{*}{$\begin{array}{l}\text { Renditen der } \\
\text { Firmenzugehörigkeit }\end{array}$} & 5 vs. 0 Jahre & $\mathbf{0 , 0 7 0} \mathbf{0}^{* * *}$ & $\mathbf{0 , 0 2 5}{ }^{* *}$ & $\mathbf{0 , 0 7 8} \mathbf{8}^{* * *}$ & $\mathbf{0 , 1 0 1} 1^{* * *}$ & $\mathbf{0 , 0 2 2} 2^{* * *}$ & $0,049^{* * *}$ \\
\hline & & $(0,014)$ & $(0,011)$ & $(0,025)$ & $(0,009)$ & $(0,007)$ & $(0,017)$ \\
\hline & 10 vs. 0 Jahre & $\mathbf{0 , 1 2 3}{ }^{* * *}$ & $\mathbf{0 , 0 4 3}{ }^{* *}$ & $0,157^{* * *}$ & $0,164^{* * *}$ & $\mathbf{0 , 0 4 9} 9^{* * *}$ & $\mathbf{0 , 0 8 2 ^ { * * }}$ \\
\hline & & $(0,019)$ & $(0,019)$ & $(0,049)$ & $(0,011)$ & $(0,012)$ & $(0,033)$ \\
\hline & 15 vs. 0 Jahre & $0,161^{* * *}$ & $\mathbf{0 , 0 5 5 ^ { * * }}$ & $\mathbf{0 , 2 2 5} 5^{* * *}$ & $0,201^{* * *}$ & $\mathbf{0 , 0 8 0 ^ { * * * }}$ & $0,096^{*}$ \\
\hline & & $(0,021)$ & $(0,027)$ & $(0,084)$ & $(0,013)$ & $(0,016)$ & $(0,056)$ \\
\hline \multirow{6}{*}{$\begin{array}{l}\text { Renditen der allg. } \\
\text { Arbeitsmarkterfahrung }\end{array}$} & 5 vs. 0 Jahre & $0,091^{* * *}$ & $\mathbf{0 , 1 1 5} 5^{* * *}$ & $\mathbf{0 , 1 2 2} * * *$ & $0,160^{* * *}$ & $\mathbf{0 , 2 0 0}{ }^{* * *}$ & $\mathbf{0 , 2 7 0}$ *** \\
\hline & & $(0,018)$ & $(0,020)$ & $(0,038)$ & $(0,014)$ & $(0,013)$ & $(0,024)$ \\
\hline & 10 vs. 0 Jahre & $\mathbf{0 , 1 4 1} 1^{* * *}$ & $\mathbf{0 , 1 8 7 ^ { * * * }}$ & $\mathbf{0 , 2 0 5} 5^{* * *}$ & $\mathbf{0 , 2 4 8} 8^{* * *}$ & $\mathbf{0 , 3 1 4} 4^{* * *}$ & $\mathbf{0 , 4 9 1 ^ { * * * }}$ \\
\hline & & $(0,025)$ & $(0,029)$ & $(0,073)$ & $(0,020)$ & $(0,019)$ & $(0,044)$ \\
\hline & 15 vs. 0 Jahre & $\mathbf{0 , 1 6 3} 3^{* * *}$ & $\mathbf{0 , 2 2 5} 5^{* * *}$ & $\mathbf{0 , 2 7 2} * *$ & $\mathbf{0 , 2 8 3}{ }^{* * *}$ & $\mathbf{0 , 3 6 6} 6^{* * *}$ & $\mathbf{0 , 6 8 2} 2^{* * *}$ \\
\hline & & $(0,027)$ & $(0,031)$ & $(0,112)$ & $(0,022)$ & $(0,020)$ & $(0,066)$ \\
\hline
\end{tabular}

Quelle: SOEP, 1996-2008. Eigene Berechnungen. ${ }^{* * *},{ }^{* *}$ und ${ }^{*}$ indizieren statistische Signifikanz auf dem 1, 5 und 10 Prozentniveau. Blockbootstrap Standardfehler sind in Klammern dargestellt

dem ostdeutschen Arbeitsmarkt tätig sind, höhere Renditen der Arbeitsmarkterfahrung erwirtschaften als ostdeutsche Arbeitskräfte. ${ }^{15}$ Nachdem sich die Renditen der westdeutschen Arbeitsmarkterfahrung nicht danach unterscheiden, in welchem regionalen Markt sie eingesetzt wird, folgern wir, dass ein reiner Preiseffekt und die Homogenität der Arbeitsmarkterfahrung unwahrscheinlich sind. Unsere Evidenz passt eher zu der Vermutung, dass die in Ostdeutschland erworbene Arbeitsmarkterfahrung von anderer Qualität ist, als die in Westdeutschland Erworbene, so dass sich daraus unterschiedlichen Lohnsteigerungen ergeben. ${ }^{16}$

\subsection{Unterschiede zwischen den Geschlechtern}

Unsere Studie analysiert zunächst die Lohnentwicklung im Lebenszyklus von Frauen und Männern im privaten Sektor (Orlowski 2011a). Da Frauen im Mittel die Erwerbstätigkeit

\footnotetext{
${ }^{15}$ Wünschenswert wäre eine getrennte Betrachtung der - nach der Wiedervereinigung gewonnenen - Arbeitsmarkterfahrung in „Ost- und Westarbeitsmarkterfahrung“. Dazu müsste man alle Personen ab 1991 fortlaufend im Datensatz beobachten, um zwischen Ost- und Westarbeitsmarkterfahrung exakt zu unterscheiden. Das ist mit den Daten des SOEP nicht möglich.

${ }^{16}$ Für eine Erklärung der flachen ostdeutschen Lohnprofile kommen auch alternative Theorien in Betracht. Bonin (2005) bietet Evidenz für die Relevanz von Lohnrigiditäten in Ostdeutschland. Acemoglu und Pischke (1999) diskutieren andere Restriktionen des Arbeitsmarktes, die zur komprimierten Lohnstrukturen und somit zu geringeren als den produktivitätsadäquaten Renditen der Arbeitsmarkterfahrung oder Firmenzugehörigkeitsdauer führen.
}

häufiger unterbrechen als Männer, verfügen sie ceteris paribus über geringere allgemeine wie firmenspezifische Arbeitsmarkterfahrung. Zusätzlich wird der unterbrochene Erwerbsverlauf von Frauen antizipiert und es ist gemäß Humankapitaltheorie (Becker 1962) plausibel zu erwarten, dass Frauen weniger in ihr Humankapital investieren als Männer, für die sich diese Investitionen bei längerer erwarteter Erwerbstätigkeit eher amortisieren. Neben diesen geschlechtsspezifischen Unterschieden in der Ausstattung mit Humankapital ist es denkbar, dass bei gleicher Ausstattung das Humankapital von Männern und Frauen aufgrund (statistischer) Diskriminierung seitens der Arbeitgeber unterschiedlich entlohnt wird.

Unsere Analysen vergleichen die Humankapitalausstattung und die Arbeitsmarktmobilität zwischen den Geschlechtern. Es zeigt sich bereits deskriptiv, dass die Anzahl der beobachten Arbeitgeberwechsel von weiblichen und männlichen Vollzeitbeschäftigten ähnlich ist und somit, entgegen der internationalen Literatur, keinen Erklärungsansatz für unterschiedliche Renditen des Humankapitals bieten kann. Ebenso ist die Ausstattung mit Humankapital für die Geschlechter ähnlich, obwohl Frauen erwartungsgemäß, durchschnittlich 1 Jahr weniger Firmenzugehörigkeit und 2 Jahre weniger allgemeine Vollzeit-Berufserfahrung haben. Im Gegenzug verfügen sie über höhere TeilzeitBerufserfahrung.

In Tab. 1 sind die Ergebnisse der Lohnschätzungen nach der KQ-Methode, nach AS (1987) und nach dem zwei- 


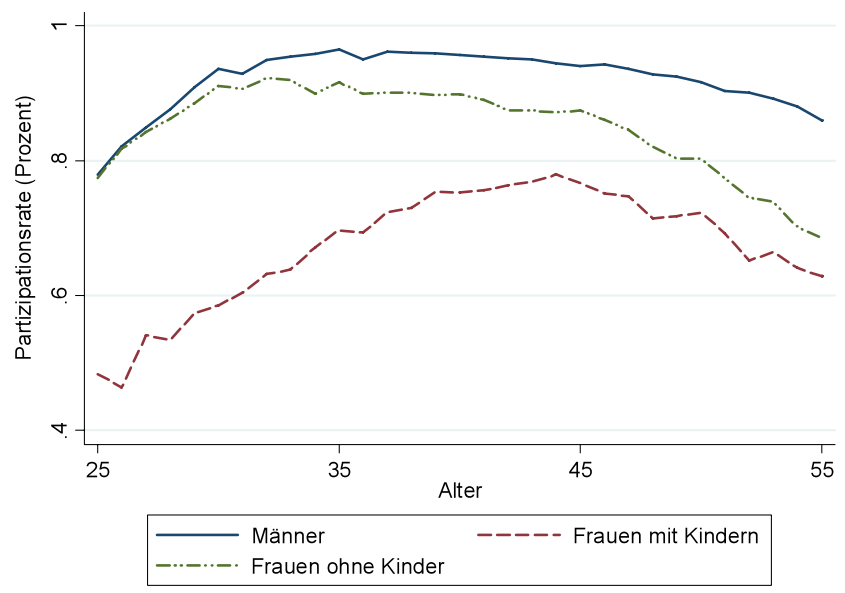

Abb. 2 Partizipationsraten von Männern und Frauen in Deutschland. Quelle: FDZ der statistischen Ämter des Bundes und der Länder, Mikrozensus 2007, eigene Berechnungen unter Verwendung von Bevölkerungsgewichten

stufigen Verfahren (2SFD) von Topel (1991) dargestellt. ${ }^{17}$ Die vorhergesagten Renditen der Firmenzugehörigkeitsdauer sind je nach Schätzmethode für Frauen niedriger (KQ), identisch (AS) und höher (2SFD) als für Männer. Somit ist kein durchgehender, systematischer Unterschied in den Renditen des firmenspezifischen Humankapitals zwischen Männern und Frauen erkennbar. Hashimoto's efficiency cost sharing Theorie findet somit keine empirische Evidenz. Allerdings sind die Renditen der allgemeinen Arbeitsmarkterfahrung für Frauen bei allen angewandten Schätzverfahren deutlich niedriger als für Männer. Wenn Frauen mehr Erwerbsunterbrechungen haben als Männer, kann dieses Ergebnis im Licht der Humankapitaltheorie mit Abschreibungen interpretiert werden. ${ }^{18}$

Der family gap Literatur folgend, untersuchen wir die Humankapitalrenditen für Frauen mit und ohne Kinder separat. Abbildung 2 zeigt die Partizipationsraten der Frauen mit und ohne Kinder. Es wird deutlich, dass Frauen ohne Kinder innerhalb des fertilen Lebens eine ähnliche Arbeitsmarktpartizipation haben wie Männer. Somit sollte die potentielle Selbstselektion in die Erwerbstätigkeit für diese Gruppe von Frauen weniger verzerrend auf Lohnschätzungen wirken als für Mütter. Die Renditen der Firmenzugehörigkeit bei Frauen ohne Kinder sind im Vergleich zu Männern kleiner (KQ, AS) oder größer (2SFD). Somit zeigen sich erneut,

\footnotetext{
${ }^{17}$ Unsere Spezifikation der Lohngleichung berücksichtigt zusätzlich das Bundesland, Jahr, Industriesektoren, Firmengröße, Wohnort vor der Wiedervereinigung, die regionale Arbeitslosenquote und den höchsten Bildungsabschluss.

${ }^{18}$ Alternative Erklärungsansätze sind mannigfaltig: Selbstselektion in bestimmte Berufe mit flachen Lohnprofilen, (statistische) Diskriminierung seitens der Arbeitgeber, diskriminierende Einstiegsgehälter, nachteilige Wiedereinstiegsmöglichkeiten oder geringere (berufliche) Weiterbildung von Müttern. Die Weiterbildung wird im SOEP nur aller vier Jahre erfasst und ist für eine weiterführende Analyse unzureichend.
}

je nach Schätzverfahren, uneindeutige Muster in den Renditen der Firmenzugehörigkeit. Hingegen sind die Renditen der Arbeitsmarkterfahrung für Frauen bei allen Schätzverfahren niedriger als bei Männern. Das Ergebnis, dass auch Frauen ohne Kinder niedrigere Renditen der Arbeitsmarkterfahrung aufweisen ist sehr interessant und könnte ein Hinweis auf statistische Diskriminierung sein. ${ }^{19}$

Abbildung 3 zeigt die Arbeitsmarktpartizipationsraten für Männer und Frauen in West- und Ostdeutschland auf Basis des Mikrozensus (2007). Es zeigt sich deutlich, dass Frauen in Ost- und Westdeutschland im Alter von 25 Jahren eine identische Partizipationsrate haben. Bereits im Alter von 35 Jahren liegt die Partizipationsrate von ostdeutschen Frauen jedoch rund 13 Prozentpunkte über der von westdeutschen Frauen und ist dem Partizipationsverhalten von ostdeutschen Männern ähnlich (für eine Enwicklung über die Zeit, vgl. Matysiak und Steinmetz 2008; Hanel und Riphahn 2010). Die empirisch geschätzten Renditen weisen die gleichen Muster auf wie bei der Analyse für Deutschland insgesamt. Allerdings sind das Lohnwachstum und die Renditen der Arbeitsmarkterfahrung sowohl bei Männern als auch bei Frauen in Ostdeutschland niedriger.

\subsection{Lohnstrukturen in großen, mittleren und kleinen Firmen}

Die Analyse der Heterogenitäten in den Lohnstrukturen in großen und kleinen Firmen berücksichtigt die Endogenität der Firmenzugehörigkeitsdauer (Orlowski 2011b). Ein wesentlicher Faktor zur Erklärung des Lohnunterschiedes könnte sein, dass große Firmen in stärkerem Maße von Tarifbindung erfasst werden als kleine Firmen. Dies reduziert die Flexibilität der Lohnsetzung. Der Grad der Tarifbindung eines Arbeitgebers wird in den Individualdaten des SOEP nicht ausreichend erfasst, deshalb wurde aus den Daten des verknüpften Arbeitgeber-Arbeitnehmer-Datensatzes LIAB des Instituts für Arbeitsmarkt- und Berufsforschung der Anteil tarifgebundener Firmen nach Jahr, Branche (Fitzenberger et al. 2011) und Region gewonnen und den Daten des SOEP zugespielt. ${ }^{20}$

Große und kleine Unternehmen sind nicht gleichmäßig zwischen Ost- und Westdeutschland verteilt. Vielfach finden sich die Unternehmenszentralen in Westdeutschland während Ostdeutschland eher von kleinen und mittelständischen

\footnotetext{
${ }^{19}$ Die Unterschiede zwischen Frauen ohne Kinder und Männern sind deutlich geringer als die Unterschiede zwischen Frauen mit Kindern und Männern. Weiterhin ist zu beachten, dass die Frauen ohne Kinder eine selektive Untergruppe aller Frauen darstellen.

${ }^{20}$ Unsere Spezifikation der Lohngleichung berücksichtigt zusätzlich das Bundesland, Jahr, Industriesektoren, Wohnort vor der Wiedervereinigung, die regionale Arbeitslosenquote und den höchsten Bildungsabschluss.
} 
Abb. 3 Partizipationsraten von Männern und Frauen in Ostund Westdeutschland. Quelle: FDZ der statistischen Ämter des Bundes und der Länder, Mikrozensus 2007, eigene Berechnungen unter Verwendung von Bevölkerungsgewichten

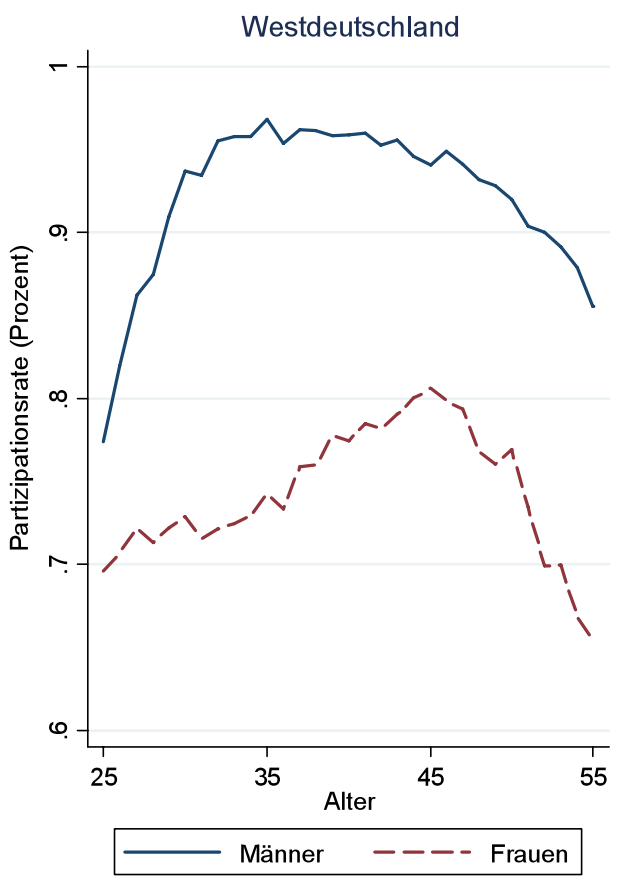

Firmen geprägt ist. Mit der unterschiedlichen Firmengrößenstruktur zwischen Ost- und Westdeutschland gehen sowohl ein unterschiedlicher Grad der Tarifbindung in den jeweiligen Branchen (Kohaut und Schnabel 2003) als auch Unterschiede in der Gewerkschaftszugehörigkeit der Arbeitnehmer zwischen Ost und West einher (Beck und Fitzenberger 2004; Fitzenberger et al. 2011).

Die vorhergesagten Renditen des Humankapitals zeigen, dass es signifikante Unterschiede in den Renditen der Firmenzugehörigkeit zwischen kleinen Firmen (bis 20 Mitarbeiter) und mittleren Firmen (bis 200 Mitarbeiter) gibt, wobei die Renditen in kleinen Firmen am niedrigsten ausfallen. Das Ergebnis lässt sich im Licht der Theorie der verzögerten Entlohnung interpretieren (Lazear 1979). In großen Firmen kommt es, verglichen mit den mittelgroßen Firmen, zu keiner weiteren Erhöhung der Renditen der Firmenzugehörigkeit. Dies kann ein Hinweis darauf sein, dass höhere Kosten der Überwachung bereits in mittelgroßen Firmen dazu führen, dass dort anstelle der Überwachung eine Motivation mittels Senioritätsrenditen erfolgt. Die Renditen der allgemeinen Arbeitsmarkterfahrung scheinen von der Firmengröße unabhängig zu sein.

\section{Schlussfolgerungen und Ausblick}

In unseren Studien analysieren wir die Lohnstrukturen in Deutschland. Der Fokus liegt auf der relativen Bedeutung der beiden Humankapitalarten Firmenzugehörigkeitsdauer und allgemeine Arbeitsmarkterfahrung und deren, unter gegebenen Annahmen, als kausal zu interpretierenden Rendi- ten bezüglich der realisierten Löhne. Wir untersuchen heterogene Lohnstrukturen in vier Dimensionen.

Es zeigt sich bei Betrachtung (a) des öffentlichen und privaten Sektors, dass sich die Humankapitalrenditen auch unter Berücksichtigung der potentiellen Endogenität des $\mathrm{Hu}$ mankapitals nicht systematisch zwischen den beiden Sektoren unterscheiden. Wir finden ähnliche Renditen der Firmenzugehörigkeit im privaten und öffentlichen Sektor. Dies gilt auch bei getrennter Analyse der Arbeitsmärkte in Ostund Westdeutschland.

(b) Im Vergleich zu westdeutschen Lohnstrukturen zeigen die Löhne am ostdeutschen Arbeitsmarkt ein extrem flaches Lohnprofil bei zunehmender allgemeiner Arbeitsmarkterfahrung, sowohl im öffentlichen wie auch im privaten Sektor. Während Erwerbstätige im Westen Deutschlands nach 10 Jahren Erwerbstätigkeit bis zu 40 Prozent Lohnsteigerung realisieren, ergeben sich für die ostdeutschen Beschäftigten hier nur Werte um 25 Prozent. Die Renditen der Firmenzugehörigkeitsdauer sind in Deutschland insgesamt niedrig und zwischen den beiden Regionen vergleichbar.

Das Ergebnis generell niedriger Renditen der Firmenzugehörigkeit ist wirtschaftspolitisch von Bedeutung. Es legt nahe, dass die hohe Arbeitslosigkeit unter älteren Arbeitnehmern vor allem in Ostdeutschland nicht durch hohe Senioritätslöhne verursacht ist, da Senioritätsentlohnung eben nicht zu beobachten ist. Auch das Resultat, dass ostdeutsche Arbeitsmarkterfahrung nur geringe Renditen erbringt, ist wirtschaftspolitisch relevant. Es legt nahe, dass die in Ostdeutschland eingesetzten Beschäftigungsmaßnahmen (Arbeitsbeschaffungsmaßnahme, ABM) zur Vermittlung von allgemeinem Humankapital kaum zu den er- 
wünschten Lohnsteigerungen führen, da Renditen des allgemeinen Humankapitals sehr gering sind. ${ }^{21}$

Die niedrigen Renditen des „ostdeutschen“ Humankapitals kann die selektive Migration nach Westdeutschland (Brücker und Trübswetter 2007) verstärken, wenn die erwarteten Renditen in Westdeutschland höher sind als die in Ostdeutschland. Dies gilt umso mehr vor dem Hintergrund der höheren Arbeitslosigkeit und geringeren Tarifbindung in Ostdeutschland und der daraus resultierende größeren Lohnsetzungsmacht der Unternehmen (Bonin 2005). Dass die Lohnstrukturen in Ost- und Westdeutschland auch knapp 20 Jahre nach der deutschen Wiedervereinigung noch nach deutlich unterschiedlichen Mechanismen bestimmt werden, lädt zu fortgesetzter wissenschaftlicher Auseinandersetzung ein.

Die Analyse der Lohnstrukturen getrennt für (c) Frauen und Männern ergeben keine systematischen Unterschiede in den Renditen der Firmenzugehörigkeit zwischen den Geschlechtern, aber interessanterweise große Unterschiede in den Renditen der allgemeinen Arbeitsmarkterfahrung. Wenn das niedrige Lohnwachstum weiblicher Beschäftigter nicht selbst ein Resultat der Erwerbsunterbrechung ist, sind die Kosten der Erwerbsunterbrechung somit gering. Falls familienbedingte Pausen zu mütterspezifischen, flachen Lohnprofilen führen, sind die Kosten der Erwerbsunterbrechung sehr hoch. Letzteres ist denkbar, wenn Arbeitgeber Mütter nicht mehr befördern (Lazear und Rosen 1990) oder weniger Weiterbildung anbieten und somit „spezielle Mütter-Karriere-Profile“ entwickeln (Miller 2009), was aber im Rahmen unserer Analysen nicht abschließend geklärt werden kann. $\mathrm{Ob}$ die gefundenen Lohnunterschiede zwischen Männern und Frauen auf geschlechterspezifische Unterschiede im Suchverhalten zurück zu führen sind, auf diskriminierende Einstiegsgehälter bei Wiedereinstieg ins Erwerbsleben, oder ob die Ergebnisse als Unterschiede im Produktivitätszuwachs über die Zeit zu interpretieren sind, ist eine spannende und noch offene Frage.

(d) Der Vergleich der Lohnstrukturen in Unternehmen unterschiedlicher Größe ergibt Unterschiede in der Rendite der Firmenzugehörigkeit. Diese fällt in kleinen Unternehmen niedriger aus. Dieses Ergebnis passt zu den Vorhersagen der deferred compensation Theorie, wonach dann Löhne oberhalb der Produktivität gezahlt werden, wenn es für den Arbeitgeber schwer ist, die Produktivität der Arbeitnehmer zu überwachen. Es ist plausibel, dass solche Überwachung in kleinen Unternehmen eher möglich ist als in Großen. Da große Unternehmen in Deutschland eher in West- als in Ostdeutschland angesiedelt sind, kann der hier festgestellte Unterschied in den Lohnstrukturen mit dazu beitragen, das be-

\footnotetext{
${ }^{21}$ Dies gilt insbesondere bei homogenen Renditen und in Hinblick auf die Erkenntnisse der Evaluationsstudien der aktiven Arbeitsmarktprogramme in Deutschland (zum Beispiel Caliendo et al. 2004).
}

obachtete Lohngefälle zwischen Ost- und Westdeutschland zu erklären.

\section{Executive summary}

This paper summarizes a group of studies investigating the determinants of German wages. The empirical analyses apply data taken from the German Socioeconomic Panel (GSOEP). The key wage determinants of interest are tenure and labor market experience. Because both might be endogenous in the estimation of wages for a number of reasons, least squares estimators likely generate biased results. Therefore, all the considered analyses additionally apply estimators developed by Altonji und Shakotko (1987) and Topel (1991). While Altonji und Shakotko (1987) proposed an instrumental variables procedure to account for the endogeneity of tenure in wage regressions Topel's procedure consists of two steps. If employees change jobs for exogenous reasons this corrects both, the endogeneity of tenure as well as of labor force participation experience. The considered studies apply the three estimators in parallel to gauge the robustness of the estimation results.

The studies focus on the heterogeneity in wage determinants in four different settings. The first setting is a comparison of wages in the public and the private sector. Here the authors are the first to consider the evidence in, both, East and West Germany. Surprisingly, the results yield no clear differences in the returns to seniority and experience between the public and private sector in East or West Germany.

The second setting focuses on a comparison of wage structures in the private sector between East and West Germany. The results indicate clear East-West differences with respect to returns to general experience. The regional heterogeneity is visible already in plots of average life-cyclewage patterns and confirmed in all estimations. In contrast, returns to tenure do not differ systematically in East versus West German wage structures. The results suggest that the different returns to experience are connected to the heterogeneity of the work experience that is gathered in East and West Germany.

The third framework for an analysis of wage structure heterogeneity compares wages for men and women. On average, observable human capital appears to be similar between men and women, even though females accumulated slightly less tenure and experience than men. Applying the different estimators yields no consistent evidence regarding different returns to tenure between the sexes. However, all estimations show lower returns to general experience for females than for men-independent of whether we stratify the samples of either sex by whether they have children, or whether they are married.

Finally, the focus is on the potential heterogeneity of wage structures across firm sizes. If monitoring problems are 
more prevalent in large companies and in the framework of Lazear's deferred compensation world this suggests higher returns to tenure in large companies. The results confirm lower returns to tenure in small (i.e. less than 20 employees) than medium sized establishments (i.e. up to 200 employees). However, the heterogeneity does not extend beyond that, as returns to tenure do not differ between medium sized and larger companies.

Overall, the contributions find evidence for heterogeneous wage structures along a number of dimensions. These results address a variety of hypotheses that are derived from theoretical models and are therefore interesting from a scientific point of view. From a social and policy perspective, it is important to know (a) that even twenty years after unification the underlying structures of the East and West German labor markets are still not comparable, and (b) that male employees are on average on career paths that generate higher returns to continued employment than females' career paths. The mechanisms driving these differences are up for further research.

\section{Deutsche Kurzfassung}

Diese Studie stellt eine Zusammenfassung mehrerer Einzelstudien dar, die sich mit den Determinanten der Löhne in Deutschland auseinandersetzen. Mit den Daten des deutschen sozioökonomischen Panels (SOEP) analysieren wir Renditen der Firmenzugehörigkeitsdauer und der allgemeinen Arbeitsmarkterfahrung. Aufgrund der potentiellen Endogenität der beiden Größen würde die klassische KleinsteQuadrate-Methode zu verzerrten Schätzergebnissen führen. Daher wenden wir zusätzlich zwei Schätzmethoden nach Altonji und Shakotko (AS, 1987) und nach Topel (1991) an. Während im Ansatz von AS Instrumentvariablen genutzt werden, wird in der Schätzmethode nach Topel ein zweistufiges Verfahren angewendet, um dem Problem der Endogenität zu begegnen. Unter der Annahme exogener Arbeitgeberwechsel, führen beide Verfahren zu unverzerrten Schätzungen der Renditen der Firmenzugehörigkeit und der allgemeinen Arbeitsmarkterfahrung. Unsere Studien berücksichtigen alle drei Schätzverfahren, um eine Vergleichbarkeit mit der bisherigen nationalen und internationalen Literatur zu gewährleisten.

Wir untersuchen die Heterogenität der Lohnstrukturdeterminanten anhand von vier Dimensionen. Dabei wird erstmals neben Westdeutschland auch der Arbeitsmarkt in Ostdeutschland berücksichtigt. In der ersten Dimension konzentrieren wir uns auf die Humankapitalrenditen im öffentlichen und privaten Sektor. Überraschendweise finden wir keine systematischen Unterschiede in Humankapitalrenditen zwischen den Sektoren. Dies gilt für Ostdeutschland und Westdeutschland.
In der zweiten Studie vergleichen wir die Lohnstrukturen im Privatsektor in Ost- und Westdeutschland. Es zeigt sich, dass die Rendite der allgemeinen Arbeitsmarkterfahrung in Ostdeutschland deutlich niedriger ist. Dieser regionale Unterschied zeigt sich bereits deskriptiv und wird in allen empirischen Schätzungen bestätigt. Im Gegensatz dazu, zeigen sich bei den Renditen der Firmenzugehörigkeit keine systematischen Unterschiede zwischen Ost und West. Die Ergebnisse legen nahe, dass die Höhe der Renditen der allgemeinen Arbeitsmarkterfahrung davon abhängen, ob die Arbeitsmarkerfahrung in Ost- oder Westdeutschland erworben wurde.

Die dritte untersuchte Dimension stellt Unterschiede in den Lohnstrukturen zwischen den Geschlechtern dar. Es zeigt sich bereits deskriptiv, dass die Humankapitalausstattung von Männern und Frauen in unserer Stichprobe im Mittel ähnlich ist. Frauen haben zwar im Mittel mehr Teilzeitarbeitsmarktfahrung, dafür aber eine leicht niedrigere Firmenzugehörigkeitsdauer und Vollzeitarbeitsmarkterfahrung. Unter Verwendung der drei Schätzmethoden zeigen wir, dass es keine Unterschiede in den Renditen der Firmenzugehörigkeit zwischen Männern und Frauen gibt. Allerdings zeigen sich systematisch niedrigere Renditen der Arbeitsmarkterfahrung für Frauen. Dieses Ergebnis ist robust, auch für die Teilstichproben verheirateter oder kinderloser Personen.

Abschließend untersuchen wir Lohnstrukturen nach Firmengröße. Wenn größere Firmen auch höhere Kosten der Überwachung haben, würde man im Modellrahmen der verzögerten Entlohnung nach Lazear, höhere Renditen der Firmenzugehörigkeit in großen Firmen erwarten. Unsere Ergebnisse bestätigen dies teilweise. Die Renditen in sehr kleinen Firmen (bis 20 Mitarbeiter) sind im Vergleich zu den Renditen in mittelgroßen Firmen (bis 200 Mitarbeiter) niedriger. Darüber hinaus steigen die Renditen der Firmenzugehörigkeit nicht weiter mit zunehmender Firmengröße an.

Unsere Studien zeigen heterogene Lohnstrukturen hinsichtlich mehrerer Dimensionen. Aus wissenschaftlicher Sicht ist dies von hohem Interesse, da viele modelltheoretisch abgeleitete Hypothesen getestet werden können. Aus sozial- und wirtschaftspolitischer Perspektive ist die Erkenntnis relevant, dass (a) auch knapp 20 Jahren nach der Wiedervereinigung, die Lohnstrukturen in Ostdeutschland nicht mit denen in Westdeutschland zu vergleichen sind und (b) dass Männer im Mittel Lohnprofile beschreiten, die ihnen höhere Renditen einbringen als die Karrierepfade der Frauen. Die Aufklärung der genauen zugrundeliegenden Mechanismen bleibt zukünftiger Forschung vorbehalten.

\section{Literatur}

Acemoglu, D., Pischke, J.S.: The structure of wages and investment in general training. J. Polit. Econ. 107(3), 539-572 (1999) 
Altonji, J.G., Blank, R.M.: Race and gender in the labor market. In: Handbook of Labor Economics. Elsevier, Amsterdam (1999)

Altonji, J.G., Shakotko, R.A.: Do wages rise with job seniority? Rev. Econ. Stud. 54, 437-459 (1987)

Altonji, J.G., Williams, N.: Do wages rise with job seniority? A reassessment. Ind. Labor Relat. Rev. 58(3), 370-397 (2005)

Amann, R.A., Klein, T.J.: Returns to type or tenure? IZA Discussion Papers No. 2773, Bonn (2007)

Bagger, J., Fontaine, F., Postel-Vinay, F., Robin, J.-M.: A feasible equilibrium search model of individual wage dynamics with experience accumulation. 2006 Meeting Papers No. 679, New York (2006)

Banzhaf, M.R.: Structural differences in job turnover and wage growth by sex and education. Ph.D. Dissertation, University of North Carolina (2005)

Beck, M., Fitzenberger, B.: Changes in union membership over time: a panel analysis for West Germany. Labour 18(3), 329-362 (2004)

Becker, E., Lindsay, C.M.: Sex differences in tenure profiles: effects of shared firm-specific investment. J. Labor Econ. 12(1), 98-118 (1994)

Becker, G.S.: Investment in human capital: a theoretical analysis. J. Polit. Econ. 70(5), 9-49 (1962)

Becker, G.S. (ed.): Human Capital, University of Chicago Press, Chicago (1975)

Berlemann, M., Thum, M.: Blooming landscapes in East Germany? CESifo Forum 6(4), 16-22 (2005)

Bird, E.J., Schwarze, J., Wagner, G.G.: Wage effects of the move toward free markets in East Germany. Ind. Labor Relat. Rev. 47(3), 390-400 (1994)

Blau, F.D., Kahn, L.M.: Rising wage inequality and the U.S. gender gap. Am. Econ. Rev. 84(2), 23-28 (1994)

BMF 2010. Ursachenanalyse der Verschiebung in der funktionalen Einkommensverteilung. Verfügbar unter: http://www. bundesfinanzministerium.de/nn_92630/DE/BMF_Startseite/ Aktuelles/Monatsbericht_des_BMF/2010/02/analysen-undberichte/b04-ursachenanalyse/ursachenanalyse.html?_nnn=true [Zugriff September 2010]

Bonin, H.: Tarifpolitik, Entgeltflexibilität und Beschäftigung in Ostdeutschland (Colletive bargaining policy, wage flexibility and employment in eastern Germany). Z Arbeitsmarktforschung (J Labour Market Res) 38(2/3), 165-179 (2005)

Boockmann, B., Steiner, V.: Cohort effects and the returns to education in West Germany. Appl. Econ. 38(10), 1135-1152 (2006)

Brown, C., Medoff, J.: The employer size-wage effect. J. Polit. Econ. 97(5), 1027-1059 (1989)

Brücker, H., Trübswetter, P.: Do the best go west? An analysis of the self-selection of East-West migrants in Germany. Empirica 34, 371-395 (2007)

Buchinsky, M., Fougère, D., Kramarz, F., Tchernis, R.: Interfirm mobility, wages and the returns to seniority and experience in the United States. Rev. Econ. Stud. 77(3), 972-1001 (2010)

Burdett, K.: A theory of employee job search and quit rates. Am. Econ. Rev. 68(1), 212-220 (1978)

Caliendo, M., Hujer, R., Thomsen, S.L.: Evaluation der Eingliederungseffekte von Arbeitsbeschaffungsmaßnahmen in reguläre Beschäftigung für Teilnehmer in Deutschland. ZEW Discussion Papers No. 04-46, Mannheim (2004)

Dustmann, C., Meghir, C.: Wages, experience and seniority. Rev. Econ. Stud. 72(1), 77-108 (2005)

Dustmann, C., Pereira, S.C.: Wage growth and job mobility in the United Kingdom and Germany. Ind. Labor Relat. Rev. 61(3), 374-393 (2008)

Dustmann, C., van Soest, A.: Wage structures in the private and public sectors in West Germany. Fisc. Stud. 18(3), 225-247 (1997)
Eickelpasch, A.: Forschung, Entwicklung und Innovationen in Ostdeutschland. Vierteljahrsh. Wirtschaftsforsch. 78(2), 78-109 (2009)

Erosa, A., Fuster, L., Restuccia, D.: Fertility decisions and gender differences in labor turnover, employment, and wages. Rev. Econ. Dyn. 5(4), 856-891 (2002)

Farber, H.S.: Mobility and stability: The dynamics of job change in labor markets. In: Orley, C.A., David, C. (eds.) Handbook of Labor Economics. Elsevier, Amsterdam (1999)

FILER, R.: The usefulness of predicted values for prior work experience in analyzing labor market outcomes for women. Journal of Human Resources, 519-537 (1993)

Fitzenberger, B., Kohn, K., Wang, Q.: The erosion of union membership in Germany: determinants, densities, decompositions. J. Popul. Econ. 24(1), 141-165 (2011)

Fitzenberger, B., Kunze, A.: Vocational training and gender: wages and occupational mobility among young workers. Oxf. Rev. Econ. Policy 21(3), 392-415 (2005)

Franz, W., Steiner, V.: Wages in the East German transition process: facts and explanations. Ger. Econ. Rev. 1(3), 241-269 (2000)

Garen, J.: The trade-off between wages and wage growth. J. Hum. Resour. 20(4), 522-539 (1985)

Gathmann, C.: The skill loss of older east germans after unification. Schmollers Jahrb. 125(1), 7-16 (2005)

Goldin, C.: Monitoring costs and occupational segregation by sex: a historical analysis. J. Labor Econ. 4(1), 1-27 (1986)

Hanel, B., Riphahn, R.T.: The employment of mothers-recent developments and their determinants in East and West Germany, CESifo Working Paper Series No. 3189, Munich (2010)

Hashimoto, M.: Firm-specific human capital as a shared investment. Am. Econ. Rev. 71(3), 475-482 (1981)

Heilemann, U.: Ostdeutschland - ein „Mezzogiorno-Fall“? Wirtschaftsdienst 85(8), 505-512 (2005)

Heitmueller, A., Mavromaras, K.G.: On the post-unification develepment of public and private pay in Germany. Manch. Sch. 75(4), 422-444 (2007)

Heywood, J.S., Jirjahn, U.: Payment schemes and gender in Germany. Ind. Labor Relat. Rev. 56(1), 44-64 (2002)

Idson, T.L., Oi, W.Y.: Workers are more productive in large firms. Am. Econ. Rev. 89(2), 104-108 (1999)

Jirjahn, U., Stephan, G.: Gender, piece rates and wages: evidence from matched employer-employee data. Camb. J. Econ. 28(5), 683-704 (2004)

Jones, D.C., Simon, I.K.: Wage determination under plan and early transition: Bulgarian evidence using matched employer-employee data. J. Comp. Econ. 33(2), 227-243 (2005)

Jovanovic, B.: Job matching and the theory of Turnover. J. Polit. Econ. 87(5), 972-990 (1979)

Jurajda, S., Harmgart, H.: When do female occupations pay more? J. Comp. Econ. 35(1), 170-187 (2007)

Kim, M.-K., Polachek, S.W.: Panel estimates of male-female earnings functions. J. Hum. Resour. 29(2), 406-428 (1994)

Kohaut, S., Schnabel, C.: Tarifverträge - nein danke!? Ausmaß und Einflussfaktoren der Tarifbindung west- und ostdeutscher Betriebe. J Econ Stat (Jahrb Nationaloekonomie Statist) 223(3), 312$331(2003)$

Kuehn, Z.: Migration, wages, and parental background: Obstacles to entrepreneurship and growth in East Germany. IMDEA Working Papers in Economics and Social Sciences No. 2010/08, Madrid (2010)

Lazear, E., Rosen, S.: Male-female wage differentials in job ladders. J. Labor Econ. 8(1), 106-123 (1990)

Lazear, E.P.: Why is there mandatory retirement? J. Polit. Econ. 87(6), 1261-1284 (1979)

Light, A., Ureta, M.: Gender differences in wages and job turnover among continuously employed workers. Am. Econ. Rev. 80(2), 293-297 (1990) 
Matysiak, A., Steinmetz, S.: Finding their way? Female employment patterns in West Germany, East Germany, and Poland. Eur. Sociol. Rev. 24(3), 331-345 (2008)

Mellow, W.: Employer size and wages. Rev. Econ. Stat. 64(3), 495501 (1982)

Melly, B.: Public-private sector wage differentials in Germany: Evidence from quantile regression. Empir. Econ. 30(2), 505-520 (2005)

Miller, A.: The effects of motherhood timing on career path. Journal of Population Economics, 1-30 (2009)

Mincer, J. (ed.): Schooling, Experience, and Earnings. National Bureau of Economic Research, New York (1974)

Mincer, J., Polachek, S.: Family investments in human capital: earnings of women. J. Polit. Econ. 82(2), 76-108 (1974)

Munasinghe, L., Reif, T., Henriques, A.: Gender gap in wage returns to job tenure and experience. Labour Econ. 15(6), 1296-1316 (2008)

Münich, D., Svejnar, J., Terrell, K.: Returns to human capital under the communist wage grid and during the transition to a market economy. Rev. Econ. Stat. 87(1), 100-123 (2005)

Noorkoiv, R., Orazem, P.F., Puur, A., Vodopivec, M.: How Estonia's economic transition affected employment and wages (19891995). Policy Research Working Paper Series No. 1837, Washington (1997)

O'Neill, J., Solomon, P.: Why the gender gap in wages narrowed in the 1980s. J Labor Econ. 11(1), 205-228 (1993)

Orlowski, R.: Gender Disparities in Returns to Tenure and Experience. Mimeo. University of Erlangen-Nuremberg, Nuremberg (2011a)

Orlowski, R.: Heterogeneity in Firm-Size Wage Structure. Mimeo. University of Erlangen-Nuremberg, Nuremberg (2011b)

Orlowski, R., Riphahn, R.T.: Seniority in Germany: new evidence on returns to tenure for full-time workers. $\mathrm{Z}$ Arbeitsmarktforschung (J Labour Mark Res) 41(2-3), 139-156 (2008)

Orlowski, R., Riphahn, R.T.: The East German wage structure after transition. Econ. Transit. 17(4), 629-659 (2009)

Pfeifer, C., Sohr, T.: Analysing the gender wage gap using personnel records of a large German company. IZA Discussion Papers No. 3533, Bonn (2008)

Polachek, S.: Occupational self-selection: a human capital approach to sex differences in occupational structure. Rev. Econ. Stat. 63(1), 60-69 (1981)

Salop, J., Salop, S.: Self-selection and turnover in the labor market. Q. J. Econ. 90(4), 619-627 (1976)
Schönberg, U.: Wage growth due to human capital accumulation and job search: a comparison between the United States and Germany. Ind. Labor Relat. Rev. 60(4), 562-586 (2007)

Statistisches Bundesamt: Pressemitteilung Nr. 311 vom 08.09.2010. Wiesbaden, Statistisches Bundesamt (2010a)

Statistisches Bundesamt: Statistisches Jahrbuch für die Bundesrepublik Deutschland. Statistisches Bundesamt, Wiesbaden (2010b) (Federal Statistical Office)

Topel, R.: Specific capital, mobility, and wages: wages rise with seniority. J. Polit. Econ. 99(1), 145-176 (1991)

Troske, K.R.: Evidence on the employer size-wage premium from worker-establishment matched data. Rev. Econ. Stat. 81(1), 1526 (1999)

Uhlig, H.: The slow decline of East Germany. J. Comp. Econ. 36(4), 517-541 (2008)

Waldfogel, J.: Understanding the "Family gap" in pay for women with children. J. Econ. Perspect. 12(1), 137-156 (1998)

Weichselbaumer, D., Winter-Ebmer, R.: A meta-analysis of the international gender wage gap. J. Econ. Surv. 19(3), 479-511 (2005)

Wellington, A.: Changes in the male/female wage gap, 1976-1985. J. Hum. Resour. 28(2), 383-411 (1993)

Williams, N.: Seniority, experience, and wages in the UK. Labour Econ. 16(3), 272-283 (2009)

Yamaguchi, S.: Job search, bargaining, and wage dynamics. J. Labor Econ. 28(3), 595-631 (2010)

Robert Orlowski After completing an apprenticeship in banking Robert Orlowski studied Economics in Munich and focused on Econometrics and Economic Policy. He received a German Diploma (M.Sc. equiv.) in Economics in 2007. In September 2007 he joined the chair of Prof. Regina T. Riphahn, Ph.D., at the University of ErlangenNuremberg.

Regina T. Riphahn earned her Ph.D. in 1995 at the University of North Carolina with a dissertation on disability retirement among German men. In 1999 she completed her Habilitation at the University of Munich. She was associate professor of economic policy at the University of Mainz (2000-2001) and of statistics and econometrics at the University of Basel (Switzerland) from 2001-2005. Since 2005 she is full professor of economics at the University of Erlangen-Nuremberg and heads the empirical economics group. 